Supporting information

\title{
Synchrotron Deep-UV Photoluminescence Imaging for the Submicrometer Analysis of Chemically Altered Zinc White Oil Paints
}

\author{
Selwin Hageraats, ${ }^{*,+\star, \S}$ Katrien Keune, ${ }^{\dagger, \S}$ Matthieu Réfrégiers, ${ }^{\perp}$ Annelies van Loon, ${ }^{\dagger}$ Barbara Berrie, ${ }^{\Uparrow}$ Mathieu Thoury ${ }^{\ddagger}$ \\ ${ }^{\dagger}$ Rijksmuseum Amsterdam, Conservation and Science, P.O. Box 74888, 1070DN Amsterdam, The Netherlands \\ ‡IPANEMA, CNRS, Ministère de la Culture, Université de Versailles Saint-Quentin-en-Yvelines, Université Paris- \\ Saclay, BP48 St. Aubin 91192 Gif-sur-Yvette, France \\ ${ }^{\S}$ Van 't Hoff Institute for Molecular Science, University of Amsterdam, P.O. Box 94157, 1090 GD Amsterdam, \\ The Netherlands \\ ${ }^{\perp}$ Synchrotron Soleil, l’Orme des Merisiers, BP48 St. Aubin 91192 Gif-sur-Yvette, France \\ "Scientific Research Department, Conservation Division, National Gallery of Art, 2000B South Club Drive, \\ Landover, MD 20785, United States \\ *E-mail: s.hageraats@rijksmuseum.nl
}

Table of contents

Deep-UV photoluminescence spectrum showing width of emission bands

Spatial correlation 327-353/412-438 nm emission images

S-2

ATR-FTIR depth profiles to exclude lead white as the main source of 327-353 and 412-438 nm luminescence 
Deep-UV photoluminescence spectrum showing width of emission bands

The average of the deep-UV-induced photoluminescence spectra recorded on the Mondrian cross-section is shown in figure S1 together with the width of some of the used emission filters. By providing a general idea of the width of the emission bands, it can be deduced from this spectrum that a major compromise in spectral resolution can be made while maintaining the essential spectral information.

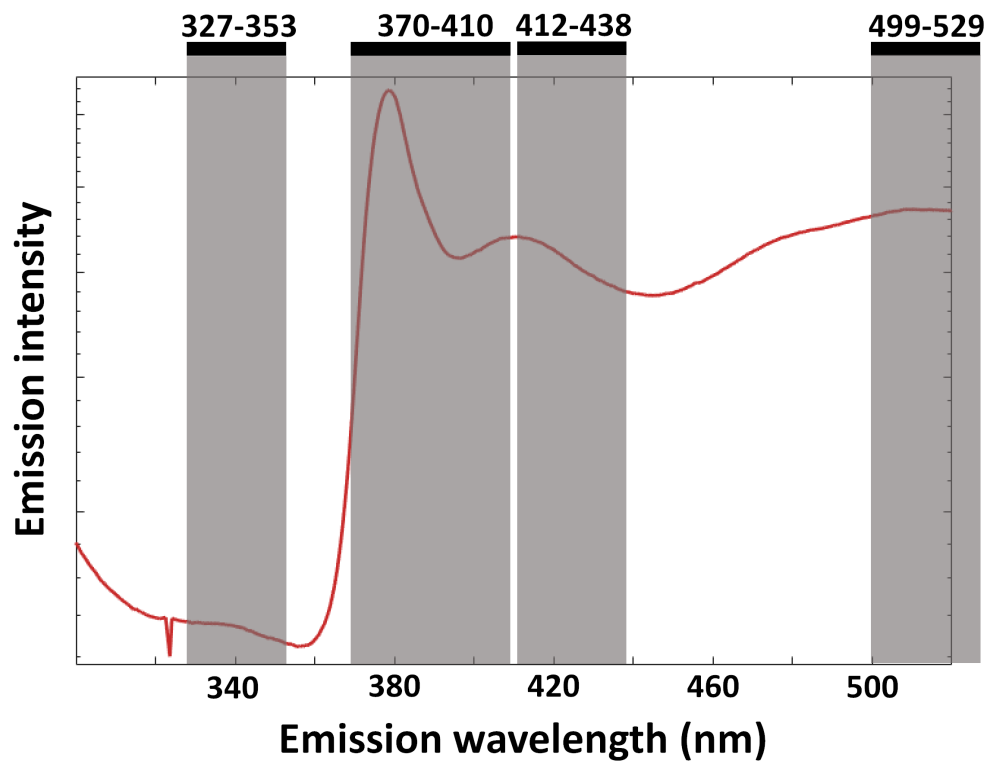

Figure S1: Average photoluminescence spectrum recorded on layers 4a, 4b, \& 5 of the Mondrian cross-section. Spectrum obtained with a $275 \mathrm{~nm}$ excitation wavelength. Grey rectangles indicate the bandwidth of four emission filters in the 300 $530 \mathrm{~nm}$ spectral range used in the multispectral acquisition approach.

Spatial correlation 327-353/412-438 $\mathrm{nm}$ emission images

From figures $3 a \& 3 c$ it is apparent that the degraded paint layer 4a exhibits luminescence in both the 327-353 and 412 $438 \mathrm{~nm}$ emission ranges. The emission in the first range could be shown to most likely originate from crystalline zinc soaps, whereas the origin of the $412-438 \mathrm{~nm}$ emission remains unclear. Based on the scale-space filtered images of layer $4 \mathrm{a}$ recorded through both emission filters (figures S2a \& S2b), it is possible to verify that both luminescence emissions are poorly spatially correlated on the microscale, and therefore have - for the most part - a different chemical origin.
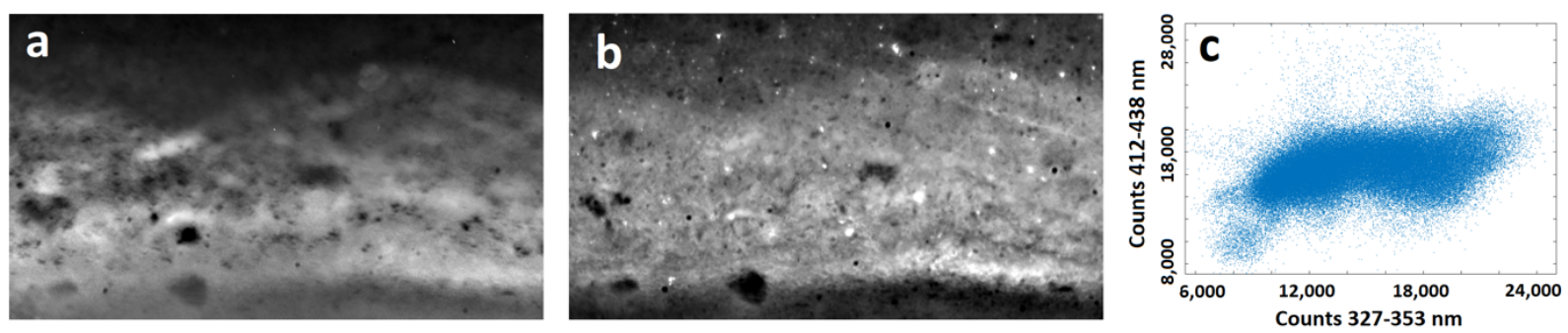

Figure S2: Structural comparison of regions of interest in the a) $327-353$ and b) $412-438 \mathrm{~nm}$ scale-space filtered photoluminescence micro-images as defined by the black rectangle in figures $3 \mathrm{a} \& 3 \mathrm{c}$. c) shows the corresponding correlation plot with Pearson correlation coefficient $\rho_{\mathrm{x}, \mathrm{y}}=0.39$. 
ATR-FTIR depth profiles to exclude lead white as the main source of 327-353 and 412-438 nm luminescence

The photoluminescence behavior of lead white is known to vary, depending for instance on the widely variable hydrocerrusite/cerrusite ratio. ${ }^{1}$ The scaled profiles of the sharp O-H stretching vibration at $3530 \mathrm{~cm}^{-1}$ (unique to hydrocerrusite) and the asymmetric C-O stretching vibration of carbonate at $1395 \mathrm{~cm}^{-1}$ (generic lead white absorption) are plotted as a function of sample depth in figure S3. The corresponding layer numbers are indicated at the top of the figure. The hydrocerrusite/cerrusite ratio inferred from figure S3 does not change between layer 4a and 4b implying that the same lead white was used in both layers. This excludes lead white as a possible candidate for producing the observed emission in the 327-353 and 412-438 nm spectral ranges, because the observed luminescence is stronger in layer 4a, even though the lead white concentration here is lower.

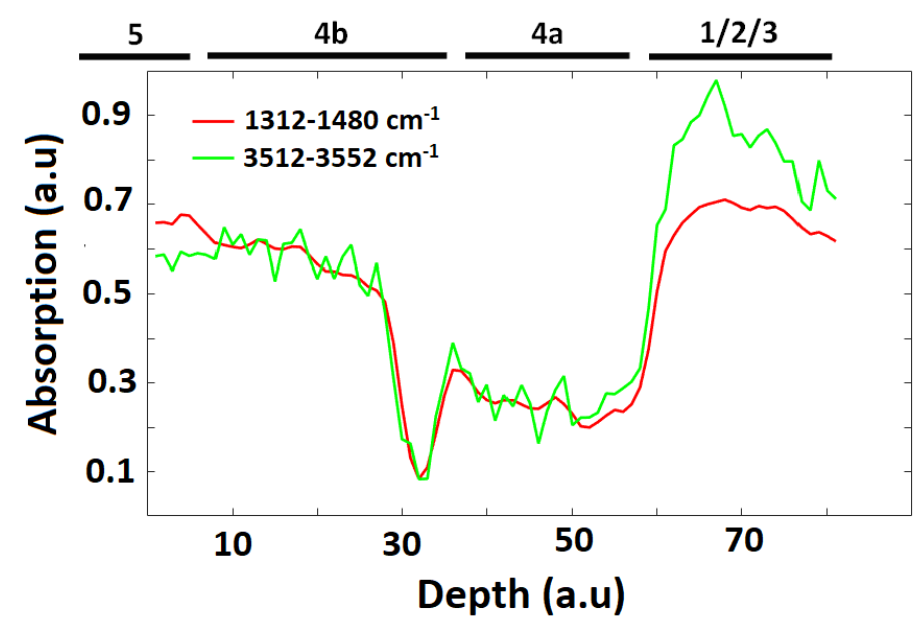

Figure S3: Integrated absorption features of lead white as function of sample depth. Values for the asymmetric C-O stretching vibration at $1395 \mathrm{~cm}^{-1}$ are shown in the red curve and represent the relative quantity of lead white in general (hydrocerrusite + cerrusite). Values for the $\mathrm{OH}$ stretch vibration at $3530 \mathrm{~cm}^{-1}$ are shown in the green curve and represent the relative quantity of hydrocerrusite. The dip in absorption is due to a surface scratch, indicated in figures 2 and 4 by a blue dashed line.

\section{References}

(1) Gonzalez, V.; Gourier, D.; Calligaro, T.; Toussaint, K.; Wallez, G.; Menu, M. Revealing the Origin and History of Lead-White Pigments by Their Photoluminescence Properties. Anal. Chem. 2017, 89, 2909-2918 